\section{PS14 A SYSTEMATIC REVIEW OF EVIDENCE ON MALIGNANT SPINAL METASTASES: TECHNOLOGIES FOR IDENTIFYING PATIENTS AT HIGH RISK OF VERTEBRAL FRACTURE AND SPINAL CORD COMPRESSION}

doi:10.1136/jech-2012-201753.113

P Suttcliffe, M Connock, D Shyangdan, R Court, N Kandala, A Clarke. Warwick Medical School, The University of Warwick, Coventry, UK

Background Spinal metastases are common and can lead to significant morbidity and reduction in quality of life towards the end of life due to spinal cord compression (SCC). Between 5\% and 20\% of patients with spinal metastases develop metastatic spinal cord compression (MSCC) during the course of their disease. Understanding early diagnosis of spinal metastases and prediction of collapse of the metastatic vertebrae is important.

Methods A systematic review was undertaken to identify patients at high risk of vertebral fracture and SCC. Thirteen electronic bibliographic databases were searched. A quality assessment instrument was used to assess bias in six domains: study population, attrition, prognostic factor measurement, outcome measurement, confounding measurement and account, and analysis.

Results 2,425 potentially relevant articles were identified, of which 31 met the inclusion criteria. Seventeen studies reported retrospective data, 10 were prospective studies, and three were other study designs. There was one systematic review. There were no randomised controlled trials. There were approximately 7,900 participants (4 studies did not provide this information) in the included studies and 5,782 participants were analysed ( 3 studies did not provide this information). The sample sizes ranged from 41 to 859. Cancers reported were: lung alone $(n=3)$; prostate alone $(n=6)$; breast alone $(n=7)$; mixed cancers $(n=13)$; and unclear $(n=1)$. Ninetythree prognostic factors were identified as potentially significant in predicting risk of SCC or collapse. Many of the included studies provided limited information about patient population and selection criteria and they varied in methodological quality, rigour and transparency. Several studies with mixed case populations identified type of cancer (e.g. breast, lung or prostate cancer) as a significant factor in predicting SCC, but determining the risk differential is difficult because of residual bias in studies.

Conclusion Perhaps not surprisingly, the overall findings from this systematic review showed that the more spinal metastases present and the longer a patient is at risk, the greater the chance of development of SCC and collapse. Other prognostic factors include total burden of metastatic disease and immediate symptomatology suggestive of spinal column involvement. Current clinical consensus favours magnetic resonance imaging and computed tomography for investigation of SCC and vertebral fracture, but this important area is clearly under-researched.

\section{PS15 POLICY ACTIONS TO REDUCE DIETARY SALT INTAKE: PRICE, PRODUCT, PLACE OR PROMOTION? A REVIEW OF THE EVIDENCE}

doi:10.1136/jech-2012-201753.114

'LC Orton, ${ }^{1} \mathrm{H}$ Bromley, ' $\mathrm{C}$ Hawkes, ${ }^{1} \mathrm{DC}$ Taylor-Robinson, 'M Moonan, 'M O'Flaherty, ${ }^{1} \mathrm{~F}$ Lloyd-Williams, ' ${ }^{2}$ M Rayner, 'S Capewell. 'Public Health \& Policy, University of Liverpool, Liverpool, UK; ${ }^{2}$ Public Health, University of Oxford, Oxford, UK

Background A range of policies aim to reduce salt intake; however, their relative effects remain unclear. We undertook a narrative synthesis of existing evidence to determine the most promising approaches.

Methods We searched for systematic reviews, then empirical and modelling studies of salt reduction policies in six electronic databases. Reference lists of retrieved articles were screened and key informants were asked to identify further reviews and empirical/ modelling studies. Items were assessed for inclusion and data were extracted into predesigned forms. Results were categorised using a modified version of the marketing four P's framework: Price, Product, Place and Promotion.

Results Price: A US sodium excise tax may reduce sodium intake (by $6 \%$ ), systolic blood pressure (by $0.9 \mathrm{mmHg}$ ), stroke (by $10 \%$ ) and myocardial infarction (by $5 \%$ ) over the lifetime of those aged $40-85$ years. (One modelling study)

Product: Voluntary US salt limits might achieve lifetime reductions in sodium intake $(-9.5 \%)$, blood pressure $(-1.25 \mathrm{mmHg})$, myocardial infarction (-5\%) and stroke (-10\%). In Australia, mandatory salt limits could reduce cardiovascular disease (by $18 \%$ ) but only by $1 \%$ with voluntary intervention. (Two modelling studies)

Promotion: Intensive advice, support and encouragement to restrict dietary sodium intake led to significant long term reductions in urinary sodium excretion, systolic blood pressure $(-1.1 \mathrm{mmHg})$ and disease burden $(-0.5 \%$.) Interventions involving advertising/marketing/labelling have only been evaluated within multi-component interventions. (One systematic review.)

Place: Place-based interventions (targeting schools, workplaces and community settings) have only been evaluated within multicomponent interventions.

Multi-component interventions: The UK FSA product and promotion-based initiatives reduced salt intake by approximately $10 \%$ Two modelling studies estimate that similar interventions in lowand middle-income countries might achieve a 15\% reduction in salt consumption, globally averting perhaps 1 million deaths and 21 million disability-adjusted life years annually. Modelling estimates from Norway suggest that combining taxes and subsidies with product and price interventions might reduce daily salt intake by 6g. Survey data reveal that in Finland between 1979 - 2002, a comprehensive and mandatory nutrition intervention (using regulations targeting price, product, place and promotion) reduced urinary sodium excretion from 13 to $10 \mathrm{~g} /$ day in men and from 10.5 to $7.5 \mathrm{~g} /$ day in women. (Two empirical studies and three modelling studies)

Conclusion There is patchy evidence on the effectiveness of policy actions to reduce dietary salt intake. Dietary advice can achieve modest benefits. Modelling suggests taxes and reformulation may be effective (particularly when mandatory). Empirical and modelling studies indicate multi-component interventions could be particularly powerful.

\section{PS16 WHICH IS MORE EFFECTIVE, A UNIVERSAL OR TARGETED APPROACH, TO IMPLEMENTING THE NATIONAL HEALTHY START PROGRAMME? A MIXED METHODS STUDY}

doi:10.1136/jech-2012-201753.115

'M Moonan, 'B Hanratty, 'M Whitehead. 'Department of Public Health and Policy, University of Liverpool, Liverpool, UK; ${ }^{2}$ Department of Health Sciences, University of York, York, UK

Background Healthy Start is a statutory scheme in the UK, aiming to improve the health of children and pregnant or lactating women by providing food vouchers and vitamins. The Department of Health intended the intervention to be targeted to low incomes families (a targeted approach), but in some areas Healthy Start vitamins are available to eligible mothers and children independent of income (a universal approach). The aim of this study is to investigate which approach is more effective and to identify barriers to implementation.

Methods 1) Systematic review of the literature to identify which approach, universal vs. targeted, achieves the highest coverage of vitamin use in pre-school children and pregnant women.; 2) Using national data, a comparison of vitamin uptake rates in targeted and universal areas.; 3) In depth qualitative interviews with 30 commissioners, providers and service users from a targeted and universal area. Data were thematically analysed. 
Results 1) Initial findings from the systematic literature review reveals that universal supplementation of vitamins such as folic acid significantly reduces the incidence of preventable ill health due to vitamin deficiencies such as neural tube defects compared to a targeted approach.; 2) In areas using the targeted approach, the uptake of children's drops and women's tablets was $1.46 \%$ and $2.56 \%$ respectively. In the area that adopted a universal approach, the uptake of children's drops and women's tablets was $3.97 \%$ and $7.72 \%$ respectively. ; 3) Barriers shared by both approaches include a lack of awareness of the scheme amongst health professionals, onerous administrative processes and the availability of vitamins. The universal approach is supported by health professionals because it does not stigmatise recipients.

Conclusion 1) From the systematic review, mandatory universal fortification of foods, e.g. flour is a major public health opportunity for the UK.; 2) Uptake of Healthy Start food vouchers and vitamins is low whatever the implementation strategy. However, uptake of Healthy Start vitamins is significantly higher in areas adopting a universal approach to implementation. A universal approach to implementation is supported by the literature and this study suggests that it may overcome some barriers to the implementation of the Healthy Start scheme nationally.; 3) In particular, a universal approach may reduce some of the administrative hurdles confronted by a targeted approach and will also address the stigma associated with the use of Healthy Start vitamins.

Producing evidence for the cost effectiveness of the scheme will be critical for its continued support.

\section{PS17 COMMISSIONING CARE FOR PEOPLE WITH LONG TERM CONDITIONS}

doi:10.1136/jech-2012-201753.116

${ }^{1}$ AM Porter, ${ }^{2} \mathrm{JA}$ Smith, ${ }^{3} \mathrm{~S}$ Shaw, ${ }^{4} \mathrm{~N}$ Mays. 'College of Medicine, Swansea University, Swansea, UK; ${ }^{2}$ Policy Department, Nuffield Trust, London, UK; ${ }^{3}$ Centre for Primary Care and Public Health, Queen Mary University of London, London, UK; ${ }^{4}$ Department of Health Services Research and Policy, LSHTM, London, UK

Background The landscape of health care commissioning is being reshaped in England, as clinical commissioning groups prepare to take on the role now held by Primary Care Trusts (PCTs). Long term conditions are likely to remain a priority area, with concerns about increasing prevalence driving a shift towards care delivered in the community and early intervention to avoid hospitalisation. We report on a NIHR-funded study which used action research in three NHS local health communities to examine in detail the practice of PCT commissioning of care for people with long term conditions. Methods The study was undertaken in three contrasting PCT areas (Somerset, Wirral and Calderdale), and focused on diabetes and another locally selected condition in each site. The design combined a largely ethnographic approach with action research, allowing for responsive intervention to meet local commissioners' needs. Formal data collection over a 15 month period to January 2012 consisted of 104 semi structured interviews, observation of 27 meetings, and analysis of over 300 documents. A thematic framework was developed to guide analysis in terms of processes, resources and outcomes of commissioning.

Results Findings highlighted the complex nature of health care commissioning, far removed from the 'commissioning cycle' which sets out a formal, sequential model emphasising contracting. Instead, commissioning developments took place over a number of years through an incremental process of review and revision where negotiation and relationships were prominent. Providers often played a significant role in identifying needs and designing new models of care. The sheer scale of labour involved in commissioning was striking. Greatest success with shifting models of care towards nationally recommended good practice came where there was a combination of effective prioritisation of developments, persistence and pragmatic bounding of tasks within a wider strategic framework. Measuring the impact of commissioning practice on clinical outcomes was challenging.

Conclusion To fulfill national policy towards remodelling care for people with long term conditions, NHS commissioners are engaged in labour-intensive, steadily paced and incremental work with providers. Planned reforms to commissioning in England raise questions about whether this approach can continue in the face of straitened budgets for management support, an increased emphasis on provider competition and disruptions to established relationships between commissioners and providers.

\section{PS18 ANALYSIS OF EMERGENCY 30-DAY READMISSIONS IN ENGLAND USING ROUTINE HOSPITAL DATA 2004-2010. IS THERE SCOPE FOR REDUCTION?}

doi:10.1136/jech-2012-201753.117

${ }^{1} \mathrm{~A}$ Clarke, ${ }^{2} \mathrm{~B}$ Blunt, ${ }^{2} \mathrm{M}$ Bardsley. ${ }^{1}$ Populations Evidence \& Technologies, Warwick Medical School, Coventry, UK; ${ }^{2}$ Research Department, Nuffield Trust, New Cavendish St, London, UK

Background A number of health systems including the NHS have recently introduced arrangements to deny payment if a patient is readmitted to hospital as an emergency soon after a period of care. These approaches assume that emergency admissions are a reflection of poor quality of care and of errors or failure in the original care episode. Our objectives were to assess the extent and types of readmission within 30 days and possible causes and scope for reduction.

Methods Retrospective analysis of 83 million routinely-collected national hospital episode statistics (HES) records covering NHS hospitals in England for a 6 year period (2004-10). Records were linked at the individual level using an annonymised person level identifier. Numbers of 30-day readmissions were calculated. We categorised readmissions using pre-defined discharge-admission diagnostic pairs, overall admission patterns and the "Bridges to Health" patient group categories.

Results There were 7,166,304 emergency 30-day readmissions over a six year period equivalent to $8.7 \%$ of all hospital discharges. Readmissions were grouped into six categories:

Potentially preventable (probable or possible suboptimal care during index admission): 1,988,967 (27.8\%);

Approach to care (anticipated but unpredictable hospital care): 1,503,282 (21.0\%);

Preference of patients or staff in admission or discharge timing: $56,514(0.8 \%)$;

Artefact in data collection: $139,508(2 \%)$;

Accident or Coincidence: 1,473,583 (20.6\%);

No obvious cause: 2,107,339 (29.4\%)

Conclusion Very large numbers of emergency readmissions fell into potentially preventable categories and to categories amenable to immediate reduction by hospitals. Denial of payment for emergency readmission has the potential to improve quality of care by improving data systems and reducing error. Action to address the majority of emergency readmissions requires assessment of care delivery across health and social care providers for those with complex, chronic or terminal conditions. In conclusion, new systems of denial of payment will be dangerous if they invoke perverse incentives which reduce access to necessary hospital care for patients.

\section{PS19 LIFETIME SOCIOECONOMIC INEQUALITIES IN PHYSICAL AND COGNITIVE AGEING}

doi:10.1136/jech-2012-201753.118

${ }^{1} \mathrm{~L}$ Hurst, ${ }^{2} \mathrm{M}$ Stafford, ${ }^{2} \mathrm{R}$ Cooper, ${ }^{2} \mathrm{M}$ Richards, ${ }^{2} \mathrm{D}$ Kuh. ${ }^{1}$ Department of Epidemiology and Public Health, UCL, London, UK; ${ }^{2}$ MRC Unit for Lifelong Health and Ageing, MRC National Survey of Health and Development, London, UK 